\title{
AVALIAÇÃO EPIDEMIOLÓGICA E RADIOLÓGICA DAS FRATURAS DIAFISÁRIAS DO FÊMUR: ESTUDO DE 200 CASOS
}

\author{
EPIDEMIOLOGICAL AND RADIOLOGICAL EVALUATION OF \\ FEMORAL SHAFT FRACTURES: STUDY OF 200 CASES
}

Frederico Barra de Moraes', Luciano Lucindo da Silva², Fábio Vieira Ferreira², Ademar Martins Ferro³, Valney Luís da Rocha ${ }^{4}$, Kim-Ir-Sen Santos Teixeira ${ }^{5}$

\section{RESUMO}

Objetivo: avaliar as características epidemiológicas e radiológicas dos casos de fratura diafisárias de fêmur, ocorridos de 1990 a 2005, tratados cirurgicamente no Hospital de Acidentados - Clínica Santa Isabel - de Goiânia, Goiás, com o propósito de contribuir para o melhor planejamento de medidas preventivas e terapêuticas a adotar em relação a essas fraturas. Métodos: Foram avaliados retrospectivamente 200 prontuários e radiografias seriadas de pacientes com fraturas diafisárias do fêmur. Não foram incluídos os pacientes com menos de 10 anos de idade, pois o tratamento para esse grupo foi conservador. Foram descartados 25 prontuários por não fornecer todos os dados necessários ao estudo. Os pacientes foram analisados quanto ao sexo, idade, lado da fratura, exposição óssea, mecanismos de trauma, classificação das fraturas, traumas associados, tempo de consolidação e tipos de fixação cirúrgica. A análise estatística foi feita pelos testes do qui-quadrado, exato de Fisher" e t de Student, considerando significância quando $\mathrm{p}$ $\leq 0,05$. Resultados: Foram significantes $(\mathrm{p} \leq 0,05)$ : em $70 \%$ de homens, $80 \%$ de fraturas fechadas e $65 \%$ de mulheres com mais de 60 anos. As fraturas por quedas da própria altura foram mais freqüentes em mulheres, acima dos 60 anos, com traços mais estáveis e as por projéteis de arma de fogo em homens, dos 20 aos 60 anos, com traços mais instáveis. Os atropelamentos predominaram entre 10 e 19 anos. Os acidentes de trânsito mostraram todos os tipos de fratura, com maior associação com outros traumas, com pico dos 20 aos 30 anos. O tratamento com hastes de Küntsher resultou em tempo de consolidação médio maior quando comparado com o da osteossíntese por haste bloqueada ou placa. Conclusão: Encontrou-se uma característica bimodal de apresentação das fraturas diafisárias do fêmur, semelhante à da literatura, onde os mecanismos de alta energia (acidentes de trânsito, quedas de alturas e ferimentos por PAF) foram mais

\section{ABSTRACT}

Objective: to evaluate epidemiological and radiological characteristics of the femoral shaft fractures, surgically treated from 1990 to 2005 at Hospital de Acidentados - Clínica Santa Isabel - in Goiânia, Goiás, aiming to contribute to better preventive and therapeutic measures planning to adopt on those fractures. Methods: 200 patients'files and x-rays with femoral shaft fractures have been retrospectively evaluated. Patients below the age of 10 years were not included because the treatment for this group was conservative. 25 files have been discarded for not supplying all the necessary data to the study. The patients were assessed for sex, age, side of the fracture, bone exposure, mechanisms of trauma, classification of the fractures, associated trauma, time for bone healing and types of surgical devices. Statistic analyses were made by chi-squared, Fisher and Student's-t tests, adopting as a significance level $p<0.05$. Results: significant results $(p<0.05)$ were found in: $70 \%$ of men, $80 \%$ closed fractures and $65 \%$ of women above the age of 60. Fractures resulting from simple falls were more frequent in women, above 60 years old, with simpler traces, and the ones caused by projectiles of firearm in men, from 20 to 60 years, with unstable traces. Trampling accidents were prevalent among youngsters between 10 and 19 years old. Car accidents showed all the types of fractures, mostly associated to other traumas, reaching its peak incidence in the age group of 20-30 years. The treatment with Küntscher Nail resulted in a longer mean consolidation time, as well as fractures with unstable traces (B3,C1,C2,C3). Conclusion: we found a bi-modal characteristic the femoral shaft fractures, consistently to literature data, where the high energy mechanisms (traffic accidents, high falls and wounds from firearms), have been more frequent in young adults,

\footnotetext{
1 - Mestre pela Universidade Federal de Goiás. Médico Ortopedista do Hospital das Clínicas da Universidade Federal de Goiás-UFG - Goiânia (GO), Brasil.

2 - Médico Estagiário do Departamento de Ortopedia e Traumatologia do Hospital das Clínicas da Universidade Federal de Goiás-UFG - Goiânia (GO), Brasil.

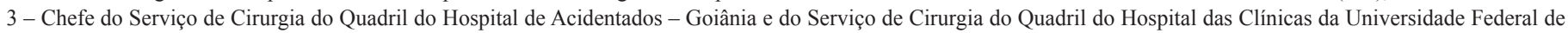
Goiás-UFG - Goiânia (GO), Brasil.

4 - Chefe do Departamento de Ortopedia e Traumatologia do Hospital das Clínicas da Universidade Federal de Goiás-UFG - Goiânia (GO), Brasil.

5 - Doutor pela Universidade Federal de Goiás.Médico Radiologista do Hospital das Clínicas da Universidade Federal de Goiás-UFG - Goiânia (GO), Brasil.
}

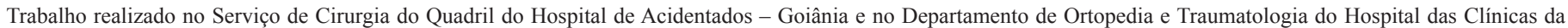
Universidade Federal de Goiás-UFG - Goiânia (GO), Brasil.

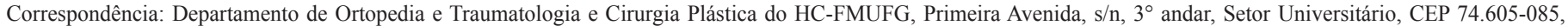
Goiânia, GO. Tel.: (62) 3269-8334. E-mail: frederico_barra@yahoo.com.br. 
frequentes em adultos jovens, sexo masculino, gerando traços instáveis de fratura, com traumas associados mais graves. O outro grupo envolvido com baixa energia (queda de própria altura) foi mais frequente em idosos, no sexo feminino, apresentando traços de fratura menos instáveis e sem traumas associados.

Descritores - Fraturas do fêmur; Diáfises; Epidemiologia; Estudos retrospectivos; Estudos de casos men, generating unstable traces of fractures, with more serious associated trauma, while the other group with low energy trauma (simple falls), was more frequently seen in elderly female individuals with less unstable fracture traces, without associated trauma.

Keywords - Femoral fractures; Diaphyses; Epidemiology; Retrospective studies; Case studies

A coleta de dados resultou da avaliação dos aspectos clínicos dos pacientes. Foram computados: sexo, idade em décadas (10 a 90) e em grupos $(10-19,20-60,>60$ anos), lado da fratura, exposição óssea ou não, mecanismos de trauma (acidentes de trânsito; quedas; ferimento por projétil de arma de fogo (PAF); traumas diretos). Foram, ainda, avaliados: lesões preexistentes (tumores malignos ou benignos), traumas associados (craniencefálicos (TCE); torácicos; fratura de outros ossos; lesões de partes moles). Foram, também, avaliados: os aspectos radiológicos (radiografias em ântero-posterior e em perfil do fêmur), o grupamento das fraturas pela classificação $\mathrm{AO}^{(7)}$; os tipos de fixação cirúrgica; o tempo de consolidação (mínimo, máximo e médio).

O tipo de fixação cirúrgica foi determinado pelo mesmo cirurgião ortopédico e levou em consideração critérios tais como: classificação da fratura, material cirúrgico disponível na cidade de Goiânia à época do atendimento, evolução técnica dos tipos de tratamento. Foram utilizados os seguintes materiais: hastes intramedulares de Küntsher, hastes intramedulares bloqueadas, placas (retas ou em ponte), sendo avaliadas as vantagens de um método em relação ao outro.

Os dados foram tabulados em programa Microsoft Excel 2000 e realizado o estudo descritivo de todas as variáveis. Na comparação estatística dos grupos de variáveis qualitativas, em grupos não pareados, foram utilizados os testes do qui-quadrado $(\chi 2)$ e o exato de Fisher, quando a frequência esperada foi menor do que cinco. Para as variáveis quantitativas, em grupos não pareados, foi utilizado o teste t de Student. Foi estabelecido como nível de significância estatística um valor igual ou menor que 0,05 ( $\mathrm{p} \leq 0,05)$.

\section{RESULTADOS}

Dos 175 casos analisados, 127 (70\%) eram homens e 48 (30\%), mulheres. Quanto ao lado, 89 (50,5\%) ocorreram no lado esquerdo e $86(49,5 \%)$ no direito, sendo 144 fraturas fechadas $(80 \%)$ e 31 expostas $(20 \%)$. As figuras 1 e 2 mostram as distribuições por faixa etária: nos pacientes com idade acima dos 60 anos, 65\% eram do sexo feminino e $35 \%$, do masculino. 


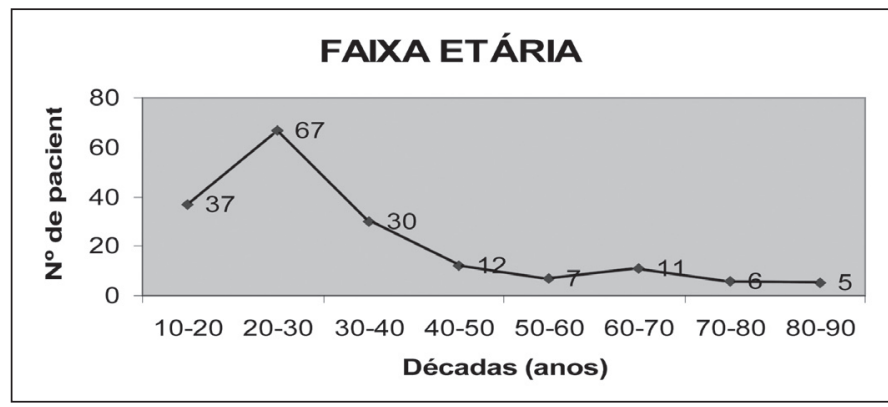

Figura 1 - Distribuição por décadas, mostrando a característica bimodal das fraturas diafisárias do fêmur (picos dos 20-30 e dos 60-70 anos)

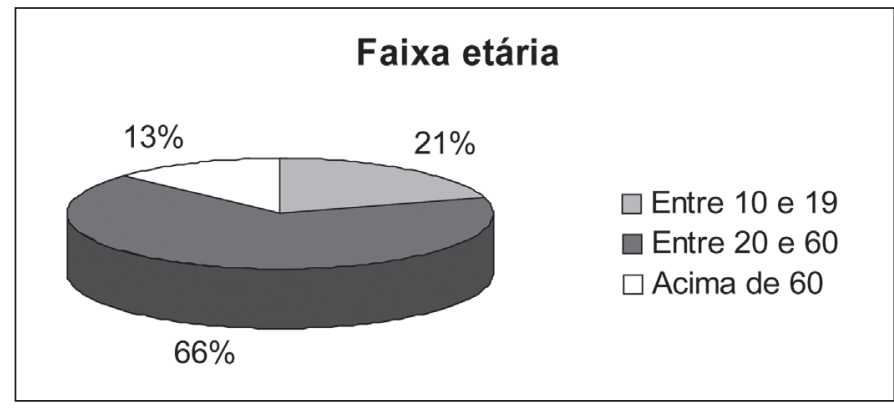

Figura 2 - Distribuição por grupos de faixas etárias específicas dos pacientes com fraturas diafisárias de fêmur

A figura 3 mostra a distribuição do sexo em relação aos mecanismos de trauma, onde as fraturas por quedas da própria altura foram mais frequentes nas mulheres $(p \leq 0,05)$ e as por PAF, em homens $(p \leq 0,05)$

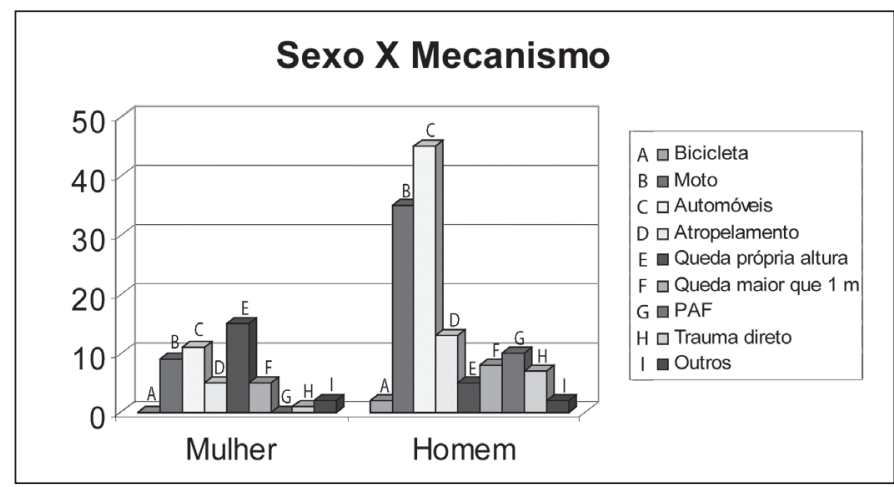

Figura 3 - Relação entre gênero e mecanismo de trauma.

A figura 4 mostra a distribuição da idade em relação aos mecanismos de trauma, onde as fraturas por quedas foram mais frequentes acima dos 60 anos $(\mathrm{p} \leq 0,05)$, as por atropelamento entre 10 e 19 anos $(p \leq 0,05)$ e as por acidente com motocicleta e automóveis, dos 20 aos 60 anos $(p \leq 0,05)$.

A figura 5 mostra a distribuição das fraturas do tipo A em relação aos mecanismos de trauma, onde as fraturas por quedas foram mais do tipo A1 $(\mathrm{p} \leq 0,05)$ e as por acidente de trânsito, do tipo A3 ( $\leq \leq 0,05)$.

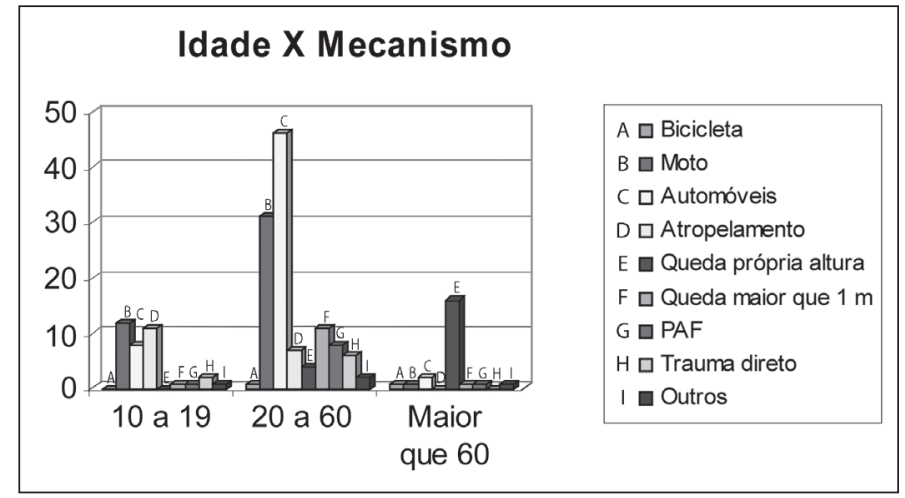

Figura 4 - Relação entre idade e mecanismo de trauma

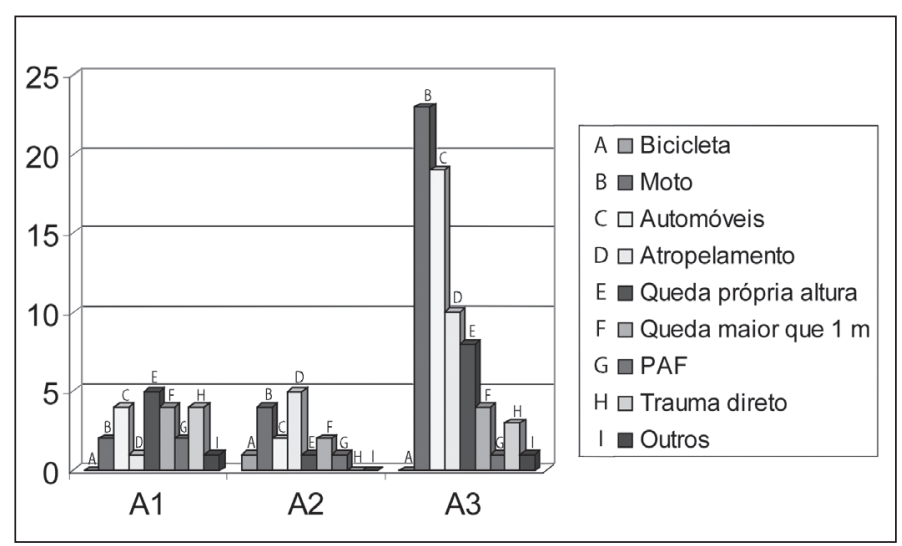

Figura 5 - Relação entre fraturas do tipo A e mecanismo de trauma.

A figura 6 mostra a distribuição das fraturas do tipo B em relação aos mecanismos de trauma, onde as fraturas por quedas predominaram no tipo $\mathrm{B} 1(\mathrm{p} \leq 0,05)$ e as por acidente de trânsito, no tipo B2 $(\mathrm{p} \leq 0,05)$.

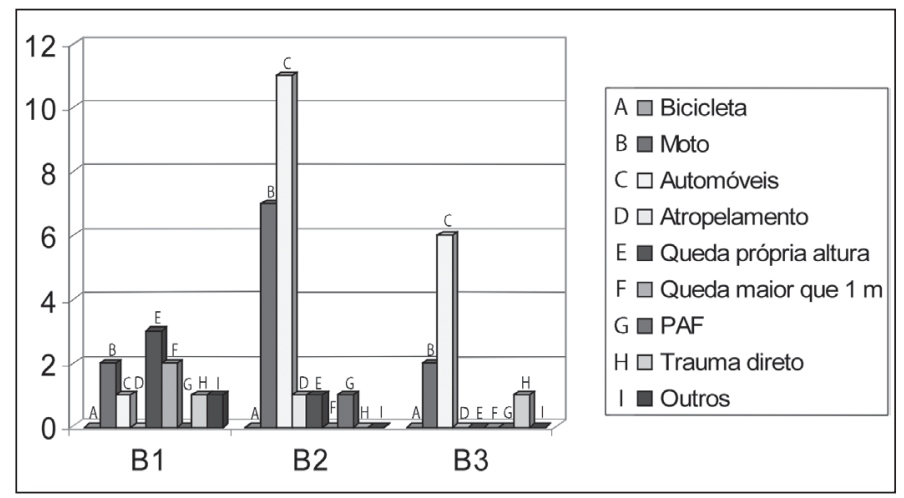

Figura 6 - Relação entre fraturas do tipo B e mecanismo de trauma.

A figura 7 mostra a distribuição das fraturas do tipo C em relação aos mecanismos de trauma, onde as fraturas dos tipos $\mathrm{C} 1$ e $\mathrm{C} 3$ predominaram nos traumatismos por PAF $(p \leq 0,05)$.

Com relação aos traumas associados às fraturas do fêmur, foram diagnosticados: TCE em 24 (13,5\%) pa- 


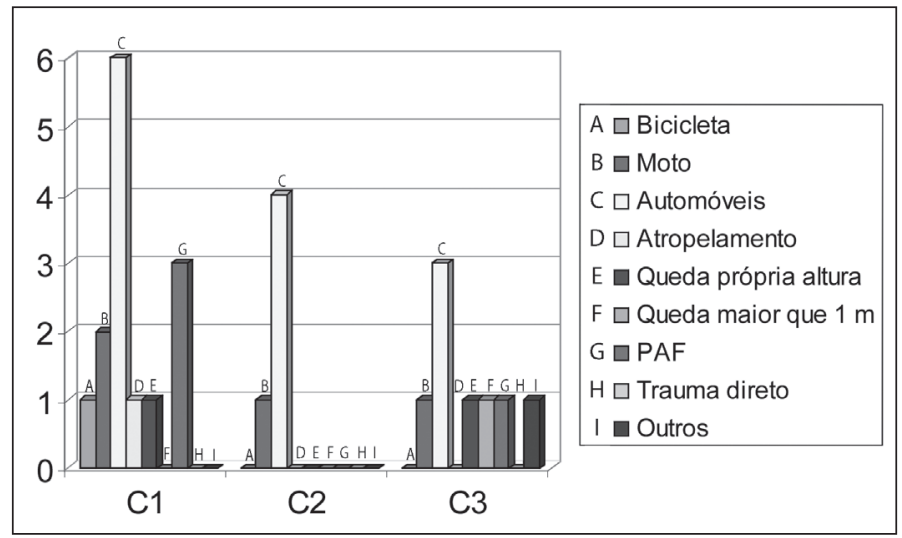

Figura 7 - Relação entre fraturas do tipo C e mecanismo de trauma.

cientes, sendo 50\% em acidentes automobilísticos, 25\% nos atropelamentos, 20,5\% com motos e 4,5\% devido à queda de ultraleve. Identificamos quatro pacientes $(2 \%)$ com trauma de tórax, três em consequência de acidente automobilístico e um de queda de ultraleve. Entre os pacientes, $23(13 \%)$ apresentavam fratura de tíbia ipsilateral: $40 \%$ em consequência de acidente automobilístico, $40 \%$ de motocicleta e $20 \%$ de atropelamentos. Detectamos 45 pacientes $(25,5 \%)$ com fraturas de outros ossos: $60 \%$ como resultado de acidente automobilístico, $20 \%$ de motocicleta, $14 \%$ por atropelamentos e $6 \%$ por queda de escada. As lesões de partes moles ocorreram em 27 pacientes $(15,5 \%)$, sendo $45 \%$ deles em consequência de acidente com motocicleta (50\% necessitando retalho cutâneo), $40 \%$ de acidente automobilístico (25\% necessitando retalho cutâneo), 11\% foram vítimas de atropelamento e $4 \%$ sofreram queda de ultraleve.

Com relação ao tempo de consolidação das fraturas, dos 175 pacientes analisados, em 52 fixações com hastes de Küntsher (30\%), a média de consolidação foi de 14 meses (3-42 meses). Em 37 fixações por hastes bloqueadas (21\%), a média foi de nove meses (4-21 meses) e em 86 placas (49\%), a média foi de oito meses (2-21 meses), sendo significante $(\mathrm{p} \leq 0,05)$ a diferença no tempo de consolidação entre os métodos com maior estabilidade (placas e hastes bloqueadas) e as hastes de Küntsher.

Ainda quanto ao tempo de consolidação, houve significância quando comparadas as fraturas mais estáveis pela classificação AO (A1 / A2 / A3 / B1 / B2), com média de nove meses, e as instáveis (B3 / C1 / C2 / C3), com média de 12 meses $(\mathrm{p} \leq 0,05)$.

\section{DISCUSSÃO}

As fraturas da diáfise femoral resultam, normalmente, de trauma de alta energia. A maioria delas ocorre em adultos jovens durante acidentes de trânsito, quedas de altura ou ferimentos por arma de fogo. Sua maior resistência deve-se à presença das forças musculares e de sustentação do peso corporal, sendo necessária força de 250 newtons/metro para fraturar a diáfise de adultos jovens ${ }^{(3)}$. O mecanismo mais comum de lesão é a carga de flexão, resultando em uma fratura transversa. Lesões de alta energia causam graus variados de cominuição da fratura.

As fraturas patológicas associadas à osteoporose ocorrem nas regiões metafisárias (osso esponjoso) ou na diáfise quando a cortical é mais fina. Ossos patológicos são propensos a fraturas espirais após pequenas cargas torcionais. Essas fraturas raramente são cominutivas ou associadas com importante dano aos tecidos moles.

As fraturas femorais diafisárias são muito frequentes no dia a dia dos ortopedistas. A mortalidade das fraturas diafisárias do fêmur se deve aos episódios de síndrome da angústia respiratória do adulto (tromboembolismo, embolia gordurosa) que ocorre em $2 \%$ dos casos, além de hemorragias e lesões arteriais. A morbidade ocorre em consequência dos encurtamentos, rotações e consolidações viciosas, infecções, rigidez articular, retardo de consolidação, pseudartrose, lesões neurais e ossificação heterotópica ${ }^{(1-3)}$.

Os tipos de mecanismos de trauma, a alta morbimortalidade e os altos gastos diretos e indiretos fazem dessas fraturas um problema de saúde pública. Estudos analisaram as características epidemiológicas dessas fraturas e encontraram uma característica bimodal bem estabelecida ${ }^{(1-6)}$.

Observamos em nosso trabalho uma característica bimodal de apresentação das fraturas diafisárias do fêmur, semelhante àquela descrita na literatura, onde os mecanismos de alta energia, como acidentes de trânsito, quedas de alturas e ferimentos por PAF, foram mais frequentes em jovens e adultos (66\%), com pico dos 20 aos 30 anos, sexo masculino (70\%), gerando traços instáveis de fratura, com traumas associados mais graves. Para esse grupo deve-se trabalhar a prevenção da violência urbana (PAF), dos acidentes de trabalho (quedas de alturas) e de trânsito, em todos os seus aspectos.

Com relação aos traumas associados às fraturas do fêmur $(25,5 \%)$, todos eles estiveram ligados aos acidentes de trânsito ou quedas de grandes alturas, pois são mecanismos de alta energia. Os acidentes envolvendo automóveis foram responsáveis por $50 \%$ desses traumas; é importante salientar que os acidentes com motocicletas originaram maior quantidade de lesões de partes moles que necessitaram retalhos (10 pacientes). Já as lesões por PAF causaram lesões graves, mas localizadas, e nas quedas de própria altura o mecanismo é de baixa energia, o que não ocasionou lesões associadas.

O outro grupo de pacientes estava envolvido em mecanismos de baixa energia, como queda de própria 
altura, mais frequente em idosos, com pico dos 60 aos 70 anos, sexo feminino (65\%), com traços de fratura menos instáveis, sem traumas associados. A prevenção dessas fraturas nesse grupo deve buscar a prevenção das quedas, por meio de programas de fortalecimento muscular para idosos, correções oftalmológicas, projetos de casas seguras, e a prevenção e tratamento da osteoporose em todas as suas etapas, na alimentação, exercícios, reposição hormonal, suplementos de cálcio e vitaminas, medicações específicas.

Quanto à classificação $\mathrm{AO}$ das fraturas, ela se mostrou útil a essa pesquisa. Observamos que os traços mais estáveis (A1, A2, A3, B1, B2) tiveram menor tempo de consolidação (nove meses) quando comparados com os traços mais instáveis (B3, C1, C2, C3) (12 meses) ( $\mathrm{p} \leq 0,05)$.

$\mathrm{O}$ tratamento das fraturas diafisárias do fêmur foi feito de forma não cirúrgica desde os tempos mais remotos, até o início do século $\mathrm{XX}^{(6-14)}$.

Atualmente, as fraturas dos tipos $\mathrm{A}$ e $\mathrm{B}$ da $\mathrm{AO}$ são preferencialmente tratadas com hastes intramedulares bloqueadas proximal e distalmente. Já as do tipo C podem ser tratadas com hastes bloqueadas ou placas em ponte. Caso o paciente esteja grave ou a lesão de partes moles seja extensa recomenda-se o uso do fixador externo no primeiro momento, trocado posteriormente para haste bloqueada ou placa em ponte. $\mathrm{O}$ tratamento com hastes de Küntscher não deve ser utilizado devido aos seus piores resultados em termos de estabilidade, bem como as placas retas com estabilidade absoluta e grandes dissecções, a não ser em localidades onde não existam os outros métodos disponíveis ${ }^{(15-28)}$.

Em nosso trabalho também observamos melhores resultados com as hastes bloqueadas e placas quanto ao tempo de consolidação, com média de nove e oito meses $(\mathrm{p} \leq 0,05)$, quando comparados com as hastes de Küntscher (14 meses), corroborando a decisão desses autores. As hastes de Küntscher foram utilizadas nos primeiros anos justamente pela falta de instrumental mais moderno disponível em nosso meio à época, bem como as placas retas de estabilidade absoluta. Atualmente, utilizamos as hastes bloqueadas e as placas em ponte.

\section{CONCLUSÕES}

Encontramos uma característica bimodal de apresentação das fraturas diafisárias do fêmur, semelhante à da literatura, onde os mecanismos de alta energia (acidentes de trânsito, quedas de altura e ferimentos por PAF) foram mais frequentes em adultos jovens, sexo masculino, gerando traços instáveis de fratura, com traumas associados mais graves. O outro grupo envolvido com baixa energia (queda de própria altura) foi mais frequente em idosos, no sexo feminino, apresentando traços de fratura menos instáveis e sem traumas associados.

\section{REFERÊNCIAS}

1. Agarwal A. Fraturas diafisárias do fêmur. In: Heckman JD, Schenck RC Jr, Agarwal A. Ortopedia - diagnóstico e tratamento. Rio de Janeiro: Guanabara-Koogan; 2001. p.88-9.

2. Brumback RJ, Ellisson TS, Poka A, Bathon GH, Burgess AR.- Intramedullary nailing of femoral shaft-fractures: Part III. Long term effects of static interlocking fixation. J Bone Joint Surg Am. 1992;74(1):106-12.

3. Bucholz RW, Brumback RJ. Fractures of the shaft of the femur. In: Rockwood CA, Green DP, Bucholz RW, Heckman JD. editors Rockwood and Green's fractures in adults. Philadelphia: Lippincott-Raven; 1996. p.1827-918.

4. Clark JD. Femur fractures: complications and treatments of traumatic femoral shaft fractures. JEMS. 2003;28(4);68-81.

5. Fernandes HJA, Reis FB, Tucci Neto PF, Belangero WD. Placa em ponte e haste intramedular bloqueada: estudo comparativo no tratamento de fraturas multifragmentárias da diáfise do fêmur. Rev Bras Ortop. 2002;37(9):392-8.

6. Zanasi R, Rotolo F, Romano P, Galmarini V, Zanasi L. Intramedullary osteosynthesis - Küntscher nailing in the femur. Ital J Orthop Traumatol. 1990;16(2):143-57.

7. Müller ME. AO Müller electronic long bone fracture classification. AO. Publishing/ thieme; 2003. Available from: URL www.aopublishing.org.

8. Adams F. The Genuine works of Hippocrates. Baltimore: Williams \& Wilkins; 1939.

9. Mathysen A. Du bandage platre et de son application dans le traitement des fractures. Liege: L. Grandmont-Donders; 1854.

10. Street D. One hundred fractures of the femur treated by mechanism of the diamondshaped medullary nail. J Bone Joint Surg Am.1951;33(3):659-78.

11. Bick EM. The intramedullary Nailing of Fractures by G. Küntscher. Translation of article in Archiv für Klinische Chirurgie, 200:443, 1940. Clin Orthop Relat Res. 1968;(60):5-12.

12. Kempf I, Grosse A, Beck G. Closed locked intramedullary nailing. J Bone Joint Surg Am. 1985;67(5):709-20.

13. Wenda K, Runkel M, Degreif J, Rudig L. Minimally invasive plate fixation in femoral shaft fractures. Injury. 1997;28(Suppl 1):13-9.

14. Warwick R; Williams PL. Gray's anatomy - Osteologia. Rio de Janeiro: Guanabara Koogan; 1979. p.339-46.

15. Bone LB, Johnson KD, Weigelt J, Scheinberg R. Early versus delayed stabilization of femo-

ral fractures. A prospective randomized study. J Bone Joint Surg Am. 1989;71(3):336-40.

16. Brumback RJ, Ellison TS Jr, Poka A, Lakatos R, Bathon GH, Burgess AR. Intramedullary nailing of open fractures of the femoral shaft. J Bone Joint Surg Am. 1989;71(9):1324-31

17. Carr $\mathrm{CR}$, Wingo $\mathrm{CH}$. Fractures of the femoral diaphysis. A retrospective study of the results and costs of treatment by intramedullary nailing and by traction and a spica cast. J Bone Joint Surg Am. 1973;55(4):690-700.

18. Chan KM, Tse PY, Chow YY, Leung PC. Closed medullary nailing for fractured shaft of the femur--a comparison between the Küntscher and the AO techniques. Injury. 1984;15(6):381-7.

19. Dabezies EJ, D'ambrosia R, Shoji H, Norris R, Murphy G. Fractures of the femoral shaft treated by extremal fixation with the Wagner device. J Bone Joint Surg Am. 1984;66(3):360-4.

20. Falavinha RS. Fixação biológica das fraturas multifragmentárias do fêmur. Rev Bras Ortop. 1996;31(6);449-56.

21. Höntzsch D. Fêmur - Diáfise. In: Rüedi TP, Murphy WM. Princípios AO do tratamento de fraturas. Porto Alegre: Artmed; 2002. p.457-67.

22. King KF, Rush J. Closed intramedullary nailing of the femoral shaft fractures. A review of one hundred and twelve cases treated by the Küntscher technique. J Bone Joint Surg Am. 1981;63(8):1319-23.

23. Paccola CAJ. Estado atual do uso de placas. Rev Bras Ortop.1994; 29(1):4-5.

24. Reeves RB; Ballard RJ, Hughes JL. Internal fixation versus traction and casting of adolescent femoral shaft fractures. J Pediatr Orthop. 1990;10(5):592-5.

25. Ruedi HP, Luscher JN. Results after internal fixation of comminuted fractures of the femoral shaft with DC plates. Clin Orthop Relat Res. 1979;(138):74-6.

26. Wagner R, Weckbach A. Complications of plate osteosynthesis of the femur shaft. An analysis of 199 femoral fractures. Unfallchirurg. 1994; 97(3):139-43.

27. Winquist RA, Hansen ST, Clawson DK. Closed intramedullary nailing of femoral fractures: a report of five hundred and twenty cases. J Bone Joint Surg Am. 1984;66(4):529-39.

28. Wright DG, Levin JS, Esterhai JL, Heppenstall RB. Immediate internal fixation of low-velocity gunshot-related femoral fractures. J Trauma. 1993;35(5):678-81. 